\title{
Reference values for biochemical analytes in feral sheep from Socorro Island, Revillagigedo Archipelago, Mexico
}

[Valores de referência para analitos bioquímicos em carneiros selvagens na Ilha Socorro, Arquipélago de Revillagigedo, México]

\author{
I.E. Arias $^{1}$, R.O.F. Prado ${ }^{2}$, M.L.J. Garcíaª, B.J.L. Zepeda ${ }^{2}$, C.A.C. García ${ }^{2 *}$
}

${ }^{1}$ Maestría en Ciencias Agropecuarias - Universidad Autónoma Metropolitana - Ciudad de México, México

${ }^{2}$ Facultad de Medicina Veterinaria y Zootecnia - Universidad de Colima - Colima, México

\begin{abstract}
To establish reference values for biochemical analytes related to freshwater shortage adaptation, a total of 376 blood samples were collected from feral sheep at Socorro Island, Revillagigedo Archipelago. Yearround variation was assessed by sampling at the beginning of each season defined by the March equinox, June solstice, September equinox, and December solstice. The resulting data set was analyzed using Gaussian distribution and descriptive statistics. Confidence intervals of $95 \%$ were established. Analysis of variance was used to compare the mean values of each season. Total cholesterol, triglycerides, urea, albumin, total protein, sodium ion, anion gap, creatine kinase, arginine vasopressin, and aldosterone showed concentrations above the reference range for domestic sheep. Triglycerides, urea, albumin, sodium ion, and aldosterone showed concentrations within the reference range for domestic goats. Most biochemical analytes showed differences $(\mathrm{P}<0.05)$ between seasons, with the highest values occurring during winter, and the lowest during spring. Results could help improve the accuracy of metabolic profiles used as a tool for evaluating dehydration indicators, and to describe the physiological mechanisms employed by feral sheep to cope with seasonal availability of freshwater.
\end{abstract}

Keywords: blood chemistry, metabolic profile, feral sheep

\section{RESUMO}

Para estabelecer valores de referência para analitos bioquímicos relacionados à adaptação da escassez. de água doce, um total de 376 amostras de sangue foram coletadas de carneiros selvagens na ilha de Socorro, no arquipélago de Revillagigedo. A variação durante todo o ano foi avaliada por amostragem no início de cada estação definida pelo equinócio de março, solstício de junho, equinócio de setembro e solstício de dezembro. O conjunto de dados resultante foi analisado usando distribuição Gaussiana e estatística descritiva. Intervalos de confiança de $95 \%$ foram estabelecidos. A análise de variância foi usada para comparar os valores médios de cada estação. O colesterol total, triglicerídeos, ureia, albumina, proteína total, íon sódio, hiato aniônico, creatina quinase, arginina vasopressina e aldosterona apresentaram concentrações acima do intervalo de referência para carneiros domésticos. Triglicerídeos, ureia, albumina, íon sódio e aldosterona apresentaram concentrações dentro da faixa de referência para cabras domésticas. A maioria dos analitos bioquímicos apresentou diferenças $(P<0,05)$ entre as estações, com os maiores valores ocorrendo no inverno e os menores na primavera. Os resultados podem ajudar a melhorar a precisão dos perfis metabólicos usados como uma ferramenta para avaliar os indicadores de desidratação e descrever os mecanismos fisiológicos empregados pelas carneiros selvagens para lidar com a disponibilidade sazonal de água doce.

Palavras-chave: bioquímica sanguínea, perfil metabólico, carneiros selvagens

Recebido em 30 de março de 2020

Aceito em 4 de agosto de 2020

*Autor para correspondência (corresponding author)

E-mail: cesargarciacasillas@hotmail.com 


\section{INTRODUCTION}

The limited availability of water for livestock has been a growing problem in some parts of the world, with droughts becoming more widespread and worsening due to climate change (Bernabucci, 2019). Consequently, it has been difficult for livestock in drought-prone areas to meet their food and fresh water needs for optimal health and production (Rust, 2019). Fortunately, small ruminants show several adaptive physiological mechanisms that may help them overcome unfavorable environmental conditions (Berihulay et al., 2019). A remarkable example of these adaptive mechanisms started with the introduction and abandonment of Merino sheep by Australian settlers to the Socorro Island of the Revillagigedo Archipelago in 1869 (Salas de León et al., 2015).

In the absence of handling, sheep became feral, adapting to the food and freshwater scarcity (Hernández et al., 2017), characteristic of volcanic islands affected by tropical storms (Ortiz et al., 2016). The prolonged isolation allowed the sheep population to develop traits adapted to unfavorable environmental conditions (Pickering et al., 2013). This chain of events provided unique traits and exceptional scientific value to the flock (Newsome, 2014). In spite of this, the Chamber of Deputies in Mexico approved the extermination of the feral sheep on the island (Ortiz et al., 2016). This measure was instrumented after findings by Evans III et al. (2015) and Ortiz et al. (2016).

These authors argued that the feral sheep of the Socorro Island contributed to deforestation by overgrazing, in addition to compacting and eroding the soil of this unique ecosystem. As a result, the Townsend's Shearwater (Puffinus auricularis) and the Socorro dove (Zenaida graysoni), endemic birds of the island, were virtually pushed to extinction. To preserve the valuable germplasm of Socorro island sheep population, the Faculty of Veterinary Medicine of the University of Colima and the National Council of Science and Technology carried out a program of extraction and recovery (Hernández et al., 2017). Regarding farm animals' endemic breeds and feral populations, several countries implement governmental programs to preserve, characterize, and assess their unique features (Ciani et al., 2015).

A thorough understanding of the adaptive physiological mechanisms in relation to limited water availability should be the starting point of all efforts to improve the resilience of domestic sheep to climate change. Thus, the aim of this study was to establish reference values for different biochemical analytes in feral sheep from Socorro Island, Revillagigedo Archipelago, Mexico. Understanding the physiological mechanisms associated to these values, would help researchers select animals that maintain their productive ability under environmental stressors such as drought.

\section{MATERIALS AND METHODS}

This experiment followed institutional and national guidelines for the care and use of animals. All experimental procedures were approved by the Committee of Ethical Review at Colima University (protocol approval number: 021/19). This study analyzed 376 blood serums obtained from the jugular vein of 94 feral sheep from Socorro Island, Revillagigedo Archipelago, Mexico. This island is located in the Pacific Ocean, $540 \mathrm{~km}$ south of Cabo San Lucas in Baja California Sur and $720 \mathrm{~km}$ west of Manzanillo, Colima. (Figure 1).

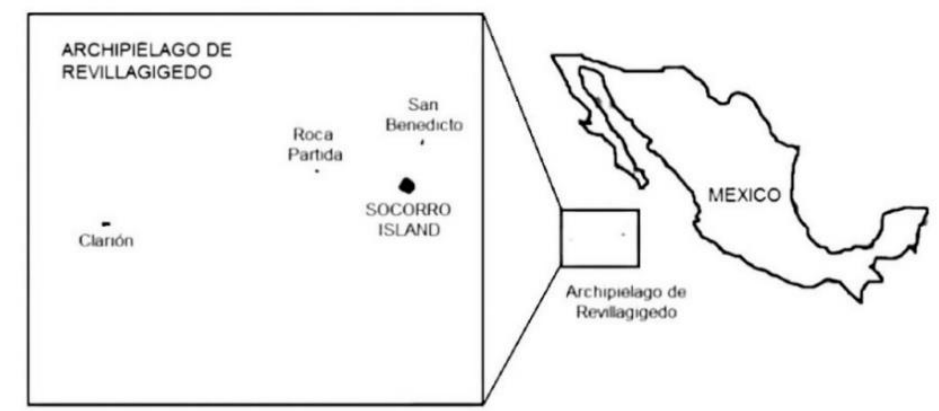

Figure 1. Location of Socorro Island, Revillagigedo Archipelago, $720 \mathrm{~km}$ west of Manzanillo, Colima 
Currently, the livestock inventory is located on the animal husbandry experimental unit in the University of Colima Faculty of Veterinary Medicine at Tecoman, Colima, Mexico ( $18^{\circ} 56^{\prime}$ $\left.53^{\prime \prime} \mathrm{N} ; 103^{\circ} 53^{\prime} 50^{\prime \prime} \mathrm{W}\right)$. Climate is warm, subhumid (Köppen $\mathrm{Cfb}$ ) with summer rains (Peel et $a l ., 2007)$. The average temperature is $26^{\circ} \mathrm{C}$ with a pluvial precipitation of $750 \mathrm{~mm} /$ year (INEGI,
2020). Sampling was done according to the methodology proposed and described by Payne et al. (1974) for the Compton metabolic profile. The total livestock inventory (94 feral sheep: 84 females and 10 males with an initial body weight [average \pm S.D.] of $45 \pm 4 \mathrm{~kg}$ and $60 \pm 6 \mathrm{~kg}$, respectively) was used (Figure 2).
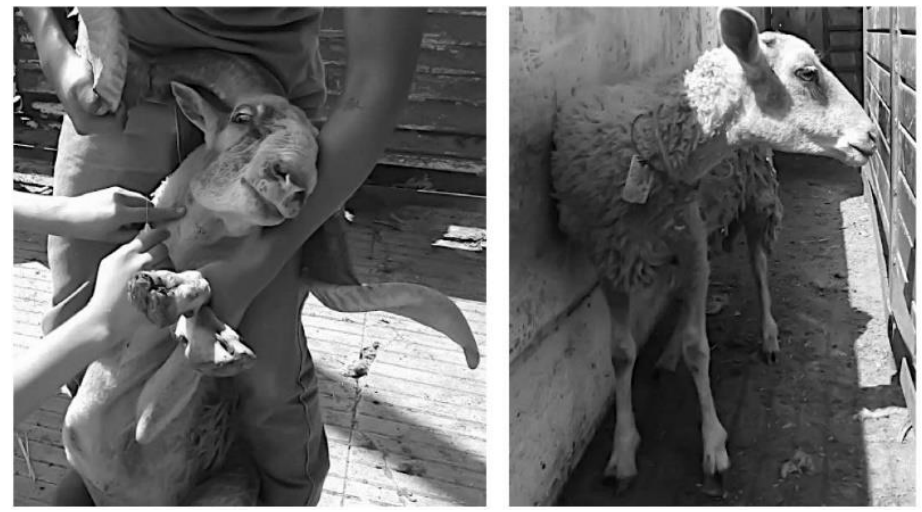

Figure 2. Feral sheep (Ovis aries) from Socorro Island, Revillagigedo Archipelago, Mexico.

To assess the annual metabolic pattern of analytes, samples were drawn from the same animals on the first day of each season of the year (solstice or equinox). Feeding conditions of sheep in the island were maintained at the experimental housing unit. Animals were able to graze Conocarpus scrub, Croton masonii scrub, and Pteridium-Dononaea scrub, introduced from Socorro Island (Flores et al., 2009). Water was available ad libitum. Before the spring sampling, the herd was dewormed with Ivermectin $(1 \mathrm{~mL} / 25 \mathrm{~kg}$ of live weight subcutaneously) followed by immunizations against Clostridium, Pasteurella multocida and Mannheimia haemolytica (BOBACT 8. SAGARPA B-0273111; Intervet., Mexico City, Mexico) 2.5mL/animal subcutaneously).

Blood samples were collected by puncture of the jugular vein before morning feeding. To analyze energy, protein, mineral, and enzyme profiles, $8.5 \mathrm{~mL}$ of blood were collected into vacuum tubes with clot activator and serum separator gel (BD Vacutainer 367988; Becton-Dickinson Co., Franklin Lakes, United States). To analyze the hormonal profile, additional samples of $5 \mathrm{~mL}$ of blood were collected into vacuum tubes with EDTA-K $_{3}$ (BD Vacutainer 366352; BectonDickinson Co., Franklin Lakes, United States). To collect serum, blood samples were centrifuged at $1500 \times \mathrm{g}$ for $10 \mathrm{~min}$ as described in Van Saun (2010) by using a portable centrifuge (Porta-Spin C828; UNICO., Dayton, United States).

Serum samples were separated using $1.5 \mathrm{~mL}$ tubes with lid (Tubes Safe-Lock 3810X; Eppendorf., Madrid, Spain) and transported at $4^{\circ} \mathrm{C}$ in a portable cooler (Thermoelectric Cooler Car/Home M5644-710; Coleman Company., Kansas, United States) to the clinical laboratories at the Autonomous Metropolitan University and the University of Colima, where they were frozen at $-20^{\circ} \mathrm{C}$ until analysis. The concentration of each analyte was determined with a UV-Vis double beam spectrophotometer (Biochemistry Analyzer; KONTROLab., Guidonia, Italy) and the hormones were determined with a gamma counter (PC-RIA MAS; Stretec., Germany). Biochemical analytes, analytical method for each parameter, units in which the results were expressed, and corresponding commercial reagents, are described in Table 1. 
Table 1. Biochemical analytes, units, analytical methods, and corresponding commercial reagents

\begin{tabular}{|c|c|c|c|}
\hline Analyte* & Unit & Method & Reagent \\
\hline \multicolumn{4}{|c|}{ Energy profile } \\
\hline Glucose (GLU) & $\mathrm{mM}$ & Colorimetric. Trinder ${ }^{a}$ & $1001190^{1}$ \\
\hline Total cholesterol (COL-T) & $\mathrm{mM}$ & Colorimetric. Liquid ${ }^{\mathrm{b}}$ & $41020^{1}$ \\
\hline Triglycerides (TAG) & $\mathrm{mM}$ & Colorimetric. Liquid ${ }^{\mathrm{c}}$ & $41032^{1}$ \\
\hline$\beta$-hydroxybutyrate $(\beta$-HBA) & $\mathrm{mM}$ & Enzymatic ${ }^{\mathrm{d}}$ & $\mathrm{RB} 1007^{2}$ \\
\hline \multicolumn{4}{|c|}{ Protein profile } \\
\hline Urea & $\mathrm{mM}$ & Enzymatic & $1001333^{1}$ \\
\hline Albumin (ALB) & $\mathrm{g} / \mathrm{dL}$ & Colorimetric. Bromocresol green & $1001020^{1}$ \\
\hline Globulin (GLOB) & $\mathrm{g} / \mathrm{dL}$ & $($ PROT-T $)-($ ALB $)$ & Difference \\
\hline Total protein (PROT-T) & $\mathrm{g} / \mathrm{dL}$ & Colorimetric. Biuret & $1001291^{1}$ \\
\hline \multicolumn{4}{|c|}{ Mineral profile } \\
\hline Calcium ion $\left(\mathrm{Ca}^{2+}\right)$ & $\mathrm{mM}$ & Colorimetric. Arsenazo III & CA2391 ${ }^{2}$ \\
\hline Inorganic phosphate $\left(\mathrm{P}_{i}\right)$ & $\mathrm{mM}$ & Colorimetric. Phosphomolybdate & $1001155^{1}$ \\
\hline Sodium ion $\left(\mathrm{Na}^{+}\right)$ & $\mathrm{mM}$ & Enzymatic. Galactosidase & $1001385^{1}$ \\
\hline Potassium ion $\left(\mathrm{K}^{+}\right)$ & $\mathrm{mM}$ & Enzymatic ${ }^{\mathrm{f}}$ & $1001395^{1}$ \\
\hline Magnesium ion $\left(\mathrm{Mg}^{2+}\right)$ & $\mathrm{mM}$ & Colorimetric. Xylidyl Blue & $1001286^{1}$ \\
\hline Chlorine ion $\left(\mathrm{Cl}^{-}\right)$ & $\mathrm{mM}$ & Colorimetric. Mercuric Thiocyanate & $1001360^{1}$ \\
\hline Carbon dioxide $\left(\mathrm{CO}_{2}\right)$ & $\mathrm{mM}$ & Enzymatic ${ }^{g}$ & $\mathrm{CD} 127^{2}$ \\
\hline Hydrogen carbonate ion $\left(\mathrm{HCO}_{3}^{-}\right)$ & $\mathrm{mM}$ & Enzymatic by $\mathrm{CO}_{2}$ total and gas dissolved & $99852^{3}$ \\
\hline Anion gap & $\mathrm{mM}$ & {$\left[\left(\mathrm{Na}^{+}+\mathrm{K}^{+}\right)-\left(\mathrm{Cl}^{-}+\mathrm{HCO}_{3}^{-}\right)\right]$} & Difference \\
\hline \multicolumn{4}{|c|}{ Enzyme profile } \\
\hline Alanine aminotransferase (ALT) & $\mathrm{U} / \mathrm{L}$ & Enzymatic $^{\mathrm{h}}$ & $41274^{1}$ \\
\hline Aspartate aminotransferase (AST) & $\mathrm{U} / \mathrm{L}$ & Enzymatic ${ }^{\mathrm{i}}$ & $41264^{1}$ \\
\hline Creatine kinase (CK) & $\mathrm{U} / \mathrm{L}$ & Enzymatic $^{j}$ & $41250^{1}$ \\
\hline$\gamma$-glutamyl transpeptidase $(\gamma$-GT) & $\mathrm{U} / \mathrm{L}$ & Enzymatic $^{\mathrm{k}}$ & $41292^{1}$ \\
\hline \multicolumn{4}{|c|}{ Hormone profile } \\
\hline Arginine vasopressin (AVP) & $\mathrm{pg} / \mathrm{mL}$ & Radioimmunoassay ${ }^{1}$ & KIPERB319 4 \\
\hline Aldosterone (Aldo) & $\mathrm{pg} / \mathrm{mL}$ & Radioimmunoassay $^{\mathrm{m}}$ & KIPZ01004 \\
\hline
\end{tabular}

*Official abbreviation of the International Union of Pure and Applied Chemistry (IUPAC); ${ }^{\text {aGlucose Oxidase- }}$ Peroxidase; ${ }^{b}$ Cholesterol Oxidase-Peroxidase; ${ }^{\mathrm{c}}$ Glycerol Phosphate Dehydrogenase-Peroxidase; ${ }^{\mathrm{d}} \beta$-hydroxybutyrate Dehydrogenase; $\quad{ }^{\mathrm{e}}$ Urease-Glutamate Dehydrogenase; ${ }^{\mathrm{f}}$ Phosphoenolpyruvate-Lactate Dehydrogenase;

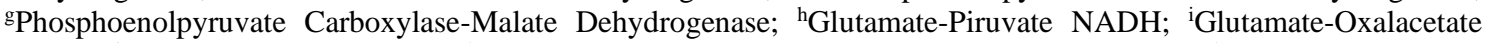
$\mathrm{NADH}$; ${ }^{\mathrm{P}}$ hosphocreatine NADPH; ${ }^{\mathrm{k}} \boldsymbol{\gamma}$-glutamyl group-Glycylglycine 2-nitro-5-aminobenzoic; ${ }^{1}$ sensitivity $20 \mathrm{pmol} / \mathrm{L}$ and intra-assay and inter-assay coefficient of variations were $5.6 \%$ and $6.1 \%$ respectively; ${ }^{\mathrm{m}}$ sensitivity $6 \mathrm{pg} / \mathrm{mL}$ and intra-assay and inter-assay coefficient of variations were $9.5 \%$ and $10.4 \%$ respectively; ${ }^{1}$ Spinreact., Girona, Spain; ${ }^{2}$ Randox Laboratories., Northern Ireland, United Kingdom; ${ }^{3}$ Biolabo Laboratory., Grandcamp-Maisy, France; ${ }^{4}$ DIAsource ImmunoAssays., Ottignies-Louvain-la-Neuve, Belgium.

The precision and reliability of the techniques were controlled using lyophilized control serum (SPINTROL NORMAL 1002100; Spinreact., Girona, Spain) and Assayed Multi-Sera AL 1027 (Randox Laboratories., Northern Ireland, United Kingdom). Hemolysis of serum was recorded on a qualitative scale of 0 (none) to 3 (dark). Samples showing hemolysis scores of 2 and above constituted less than $1 \%$ of all samples and did not introduce a significant bias in any of the tested models; thus, the influence of serum hemolysis was ignored.

The statistical procedure used to calculate $95 \%$ confidence intervals for the different biochemical analytes follows the recommendation of the
International Federation of Clinical Chemistry (Solberg, 1987). The data were described by means and SD and they were tested for normal distribution (Shapiro-Wilk test). The comparison between seasons was assessed by Analysis of Variance (SPSS..., 2013). A multiple comparison test of Tukey was performed when the effect of season was found to be significant $(\mathrm{P}<0.05)$.

\section{RESULTS}

The descriptive statistics for glucose (GLU), total cholesterol (COL-T), triglycerides (TAG), $\beta$ hydroxybutyrate ( $\beta$-HBA), urea, albumin (ALB), globulin (GLOB), total protein (PROT-T), calcium ion $\left(\mathrm{Ca}^{2+}\right)$, inorganic phosphate $\left(\mathrm{P}_{i}\right)$, 
sodium ion $\left(\mathrm{Na}^{+}\right)$, potassium ion $\left(\mathrm{K}^{+}\right)$, magnesium ion $\left(\mathrm{Mg}^{2+}\right)$, chloride ion $\left(\mathrm{Cl}^{-}\right)$, carbon dioxide $\left(\mathrm{CO}_{2}\right)$, hydrogen carbonate ion $\left(\mathrm{HCO}_{3}^{-}\right)$, anion gap, alanine aminotransferase (ALT), aspartate aminotransferase (AST), creatine kinase $(\mathrm{CK}), \gamma$-glutamyl transpeptidase $(\gamma-\mathrm{GT})$, arginine vasopressin or antidiuretic hormone (AVP), and aldosterone (Aldo), determined from 376 blood serums of 94 feral sheep from Socorro Island, Revillagigedo Archipelago, and its respective international reference range values for small ruminants (sheep and goat), are shown in Table 2.

The energy profile: COL-T and TAG showed concentrations above the reference range for sheep. The TAG results were within the reference value for goats. The protein profile: urea, ALB, and PROT-T showed higher concentrations than the reference range for sheep. The urea and ALB levels were within the reference value for goats. The mineral profile: $\mathrm{Na}^{+}$and anion gap concentrations were above the reference range for sheep. $\mathrm{Na}^{+}$measures were within the reference value for goats. The enzyme profile: CK concentration was slightly above the reference range for sheep, and the hormone profile: AVP and Aldo concentrations were above the reference range for sheep. The Aldo showed levels within the reference value for goats (Table 2).

Table 2. Mean \pm standard deviation, reference range value, and confidence interval for different biochemical analytes of feral sheep from Socorro Island, Revillagigedo Archipelago, by the annual metabolic scheme. Combined males and females $n=376$ blood serums

\begin{tabular}{|c|c|c|c|c|}
\hline Analyte & $\begin{array}{c}\text { Mean } \pm \text { standard } \\
\text { deviation }\end{array}$ & $\begin{array}{l}\text { Reference range } \\
\text { for sheep }\end{array}$ & $\begin{array}{l}\text { Reference range } \\
\text { for goat }\end{array}$ & $\begin{array}{c}\text { Confidence } \\
\text { interval of } 95 \%\end{array}$ \\
\hline \multicolumn{5}{|l|}{ Energy profile } \\
\hline Glucose $(\mathrm{mM})$ & $3.76 \pm 0.25$ & $3.80 \pm 0.33^{\dagger}$ & $3.49 \pm 0.39^{\dagger}$ & $3.73-3.78$ \\
\hline Total cholesterol $(\mathrm{mM})^{* *}$ & $2.14 \pm 0.12$ & $1.66 \pm 0.31^{\dagger}$ & $1.55 \pm 0.67^{\dagger}$ & $2.13-2.15$ \\
\hline Triglycerides $(\mathrm{mM})^{*}$ & $0.39 \pm 0.01$ & $0.33 \pm 0.01^{\S}$ & $0.20 \pm 0.09^{¥}$ & $0.39-0.39$ \\
\hline$\beta$-hydroxybutyrate (mM) & $0.59 \pm 0.01$ & $0.55 \pm 0.04^{\dagger}$ & $0.50 \pm 0.14^{¥}$ & $0.59-0.59$ \\
\hline \multicolumn{5}{|l|}{ Protein profile } \\
\hline Urea $(\mathrm{mM}) * *$ & $8.63 \pm 0.39$ & $7.70 \pm 0.43^{\S}$ & $9.60 \pm 3.02^{¥}$ & $8.59-8.67$ \\
\hline Albumin $(\mathrm{g} / \mathrm{dL}) * *$ & $3.53 \pm 0.39$ & $2.70 \pm 0.11^{\dagger}$ & $3.30 \pm 0.33^{\dagger}$ & $3.49-3.57$ \\
\hline Globulin $(\mathrm{g} / \mathrm{dL})$ & $5.19 \pm 0.09$ & $4.40 \pm 0.73^{\dagger}$ & $3.60 \pm 0.50^{\dagger}$ & $5.18-5.20$ \\
\hline Total protein $(\mathrm{g} / \mathrm{dL})^{*}$ & $8.73 \pm 0.34$ & $7.20 \pm 0.52^{\dagger}$ & $6.90 \pm 0.48^{\dagger}$ & $8.69-8.76$ \\
\hline \multicolumn{5}{|l|}{ Mineral profile } \\
\hline Calcium ion $(\mathrm{mM})$ & $3.06 \pm 0.12$ & $3.04 \pm 0.07^{\dagger}$ & $2.58 \pm 0.18^{\dagger}$ & $3.05-3.08$ \\
\hline Inorganic phosphate (mM) & $2.12 \pm 0.16$ & $2.07 \pm 0.06^{\dagger}$ & $4.62 \pm 0.25^{\dagger}$ & $2.10-2.14$ \\
\hline Sodium ion $(\mathrm{mM})^{* *}$ & $162.9 \pm 11.4$ & $141.5 \pm 4.1^{\dagger}$ & $150.0 \pm 3.1^{\dagger}$ & $161.7-164.1$ \\
\hline Potassium ion (mM) & $4.32 \pm 0.20$ & $4.23 \pm 0.5^{\S}$ & $4.40 \pm 0.56^{¥}$ & $4.30-4.34$ \\
\hline Magnesium ion (mM) & $1.18 \pm 0.12$ & $1.03 \pm 0.12^{\dagger}$ & $1.32 \pm 0.14^{\dagger}$ & $1.17-1.19$ \\
\hline Chlorine ion (mM) & $99.04 \pm 2.70$ & $99.0 \pm 4.0^{\dagger}$ & $105.1 \pm 2.9^{\dagger}$ & $98.7-99.3$ \\
\hline Carbon dioxide (mM) & $26.79 \pm 1.27$ & $26.20 \pm 1.0^{\dagger}$ & $27.40 \pm 1.40^{\dagger}$ & $26.66-26.92$ \\
\hline Hydrogen carbonate ion (mM) & $23.29 \pm 1.24$ & $22.50 \pm 2.50^{\dagger}$ & $26.53 \pm 2.66^{\infty}$ & $23.17-23.42$ \\
\hline Anion gap $(\mathrm{mM}) *$ & $44.92 \pm 11.83$ & $24.23 \pm 3.90^{\circ}$ & $22.87 \pm 3.70^{\circ}$ & $43.72-46.11$ \\
\hline \multicolumn{5}{|l|}{ Enzyme profile } \\
\hline $\begin{array}{l}\text { Alanine aminotransferase } \\
\text { (U/L) }\end{array}$ & $26.77 \pm 2.10$ & $24.13 \pm 1.0^{\S}$ & $12.0 \pm 6.0^{\dagger}$ & $26.56-26.98$ \\
\hline $\begin{array}{l}\text { Aspartate aminotransferase } \\
\text { (U/L) }\end{array}$ & $79.87 \pm 2.11$ & $80.92 \pm 4.0^{\S}$ & $85.1 \pm 28.02^{¥}$ & $79.65-80.08$ \\
\hline \multirow{2}{*}{$\begin{array}{l}\text { Creatine kinase (U/L)* } \\
\gamma \text {-glutamyl transpeptidase } \\
\text { (U/L) }\end{array}$} & $13.07 \pm 0.26$ & $10.3 \pm 1.6^{\dagger}$ & $4.50 \pm 2.8^{\dagger}$ & $13.04-13.10$ \\
\hline & $35.88 \pm 1.42$ & $33.50 \pm 4.30^{\dagger}$ & $38.0 \pm 13.0^{\dagger}$ & $35.73-36.02$ \\
\hline \multicolumn{5}{|l|}{ Hormone profile } \\
\hline Arginine vasopressin $(\mathrm{pg} / \mathrm{mL}) *$ & $8.29 \pm 0.40$ & $3.30 \pm 1.37^{\mathfrak{x}}$ & $13.64 \pm 1.72^{£}$ & $8.25-8.34$ \\
\hline Aldosterone $(\mathrm{pg} / \mathrm{mL}) * *$ & $27.86 \pm 1.03$ & $20.10 \pm 2.21^{\mathbb{I I}}$ & $30.10 \pm 2.21^{\mathfrak{x}}$ & $27.75-27.96$ \\
\hline
\end{tabular}

$\bar{\dagger}$ (Kaneko et al., 2008); ${ }^{\S}\left(\right.$ Lotfollahzadeh et al., 2016); ${ }^{\ddagger}$ (Soares et al., 2018); ${ }^{\infty}$ (Antunović et al., 2019); ${ }^{\mathfrak{x}}$ (Mengistu et al., 2016); ${ }^{£}\left(\right.$ Kaliber et al., 2016); II(Kataria and Kataria, 2007); ${ }^{\circ}$ International reference range (sodium ion + potassium ion) - (chlorine ion + hydrogen carbonate ion)]; *Differences with the reference range for sheep. 
As shown in Table 3, except for GLU, TAG, $\mathrm{Na}^{+}$, $\mathrm{CO}_{2}$, and $\gamma-\mathrm{GT}$, the serum concentration of all other biochemical analytes showed differences $(P<0.05)$, between seasons. Higher values correspond to the first day of winter (December solstice), and lowest values to the first day of spring (March equinox).

Table 3. Comparison of different biochemical analytes of feral sheep from Socorro Island, Revillagigedo Archipelago. Combined males and females $n=94$ blood serums/group

\begin{tabular}{|c|c|c|c|c|}
\hline Analyte* $^{*}$ & March equinox & June solstice & $\begin{array}{c}\text { September } \\
\text { equinox }\end{array}$ & $\begin{array}{c}\text { December } \\
\text { solstice }\end{array}$ \\
\hline \multicolumn{5}{|l|}{ Energy profile } \\
\hline Glucose $(\mathrm{mM})$ & $3.75 \pm 0.24^{\mathrm{a}}$ & $3.76 \pm 0.25^{\mathrm{a}}$ & $3.75 \pm 0.24^{\mathrm{a}}$ & $3.76 \pm 0.26^{\mathrm{a}}$ \\
\hline Total cholesterol (mM) & $2.08 \pm 0.11^{\mathrm{a}}$ & $2.13 \pm 0.11^{\mathrm{b}}$ & $2.13 \pm 0.11^{\mathrm{b}}$ & $2.22 \pm 0.11^{\mathrm{c}}$ \\
\hline Triglycerides $(\mathrm{mM})$ & $0.39 \pm 0.01^{\mathrm{a}}$ & $0.39 \pm 0.01^{\mathrm{a}}$ & $0.39 \pm 0.02^{\mathrm{a}}$ & $0.39 \pm 0.01^{\mathrm{a}}$ \\
\hline$\beta$-hydroxybutyrate (mM) & $0.58 \pm 0.012^{\mathrm{a}}$ & $0.58 \pm 0.011^{\mathrm{b}}$ & $0.59 \pm 0.012^{\mathrm{b}}$ & $0.60 \pm 0.011^{\mathrm{c}}$ \\
\hline \multicolumn{5}{|l|}{ Protein profile } \\
\hline Urea $(\mathrm{mM})$ & $8.15 \pm 0.18^{\mathrm{a}}$ & $8.51 \pm 0.14^{\mathrm{b}}$ & $8.72 \pm 0.12^{\mathrm{c}}$ & $9.15 \pm 0.17^{\mathrm{d}}$ \\
\hline Albumin (g/dL) & $3.04 \pm 0.16^{\mathrm{a}}$ & $3.42 \pm 0.10^{\mathrm{b}}$ & $3.63 \pm 0.08^{c}$ & $4.04 \pm 0.22^{\mathrm{d}}$ \\
\hline Globulin (g/dL) & $5.25 \pm 0.06^{\mathrm{a}}$ & $5.19 \pm 0.04^{b}$ & $5.21 \pm 0.04^{b}$ & $5.11 \pm 0.11^{\mathrm{c}}$ \\
\hline Total protein $(\mathrm{g} / \mathrm{dL})$ & $8.30 \pm 0.15^{\mathrm{a}}$ & $8.62 \pm 0.14^{b}$ & $8.84 \pm 0.12^{\mathrm{c}}$ & $9.16 \pm 0.14^{\mathrm{d}}$ \\
\hline \multicolumn{5}{|l|}{ Mineral profile } \\
\hline Calcium ion $(\mathrm{mM})$ & $2.90 \pm 0.05^{\mathrm{a}}$ & $3.02 \pm 0.03^{b}$ & $3.11 \pm 0.02^{\mathrm{c}}$ & $3.22 \pm 0.05^{\mathrm{d}}$ \\
\hline Inorganic phosphate (mM) & $2.06 \pm 0.11^{\mathrm{a}}$ & $2.11 \pm 0.11^{\mathrm{b}}$ & $2.12 \pm 0.11^{\mathrm{b}}$ & $2.21 \pm 0.11^{\mathrm{c}}$ \\
\hline Sodium ion (mM) & $161.28 \pm 11.53^{\mathrm{a}}$ & $\begin{array}{c}163.18 \pm \\
11.24^{\mathrm{a}}\end{array}$ & $162.32 \pm 12.01^{\mathrm{a}}$ & $164.98 \pm 11.04^{\mathrm{a}}$ \\
\hline Potassium ion (mM) & $4.10 \pm 0.15^{\mathrm{a}}$ & $4.32 \pm 0.11^{\mathrm{b}}$ & $4.33 \pm 0.12^{\mathrm{b}}$ & $4.54 \pm 0.13^{c}$ \\
\hline Magnesium ion (mM) & $1.14 \pm 0.12^{\mathrm{a}}$ & $1.18 \pm 0.11^{\mathrm{a}}$ & $1.18 \pm 0.12^{\mathrm{a}}$ & $1.23 \pm 0.11^{\mathrm{b}}$ \\
\hline Chlorine ion (mM) & $95.77 \pm 2.07^{\mathrm{a}}$ & $98.40 \pm 0.50^{\mathrm{b}}$ & $99.66 \pm 0.53^{\mathrm{c}}$ & $102.34 \pm 1.41^{\mathrm{d}}$ \\
\hline Carbon dioxide (mM) & $26.79 \pm 1.29^{\mathrm{a}}$ & $26.85 \pm 1.25^{\mathrm{a}}$ & $26.70 \pm 1.34^{\mathrm{a}}$ & $26.83 \pm 1.23^{\mathrm{a}}$ \\
\hline Hydrogen carbonate ion (mM) & $21.72 \pm 0.62^{\mathrm{a}}$ & $22.87 \pm 0.21^{\mathrm{b}}$ & $23.69 \pm 0.19^{c}$ & $24.90 \pm 0.57^{\mathrm{d}}$ \\
\hline Anion gap $(\mathrm{mM})$ & $47.88 \pm 11.81^{\mathrm{a}}$ & $46.22 \pm 11.32^{\mathrm{ab}}$ & $43.30 \pm 12.24^{\mathrm{b}}$ & $42.28 \pm 11.25^{\mathrm{b}}$ \\
\hline \multicolumn{5}{|l|}{ Enzyme profile } \\
\hline Alanine aminotransferase (U/L) & $26.93 \pm 1.95^{\mathrm{a}}$ & $27.23 \pm 2.33^{\mathrm{a}}$ & $26.98 \pm 2.13^{\mathrm{a}}$ & $25.96 \pm 1.76^{\mathrm{b}}$ \\
\hline Aspartate aminotransferase (U/L) & $80.09 \pm 1.95^{\mathrm{a}}$ & $80.32 \pm 2.32^{\mathrm{a}}$ & $80.09 \pm 2.13^{\mathrm{a}}$ & $78.97 \pm 1.79^{b}$ \\
\hline Creatine kinase $(\mathrm{U} / \mathrm{L})$ & $12.72 \pm 0.09^{\mathrm{a}}$ & $13.01 \pm 0.04^{\mathrm{b}}$ & $13.14 \pm 0.05^{\mathrm{c}}$ & $13.42 \pm 0.10^{\mathrm{d}}$ \\
\hline$\gamma$-glutamyl transpeptidase (U/L) & $35.94 \pm 1.45^{\mathrm{a}}$ & $35.87 \pm 1.42^{\mathrm{a}}$ & $35.87 \pm 1.41^{\mathrm{a}}$ & $35.84 \pm 1.43^{\mathrm{a}}$ \\
\hline \multicolumn{5}{|l|}{ Hormone profile } \\
\hline Arginine vasopressin $(\mathrm{pg} / \mathrm{mL})$ & $8.13 \pm 0.39^{\mathrm{a}}$ & $8.28 \pm 0.37^{b}$ & $8.29 \pm 0.39^{b}$ & $8.47 \pm 0.37^{c}$ \\
\hline Aldosterone $(\mathrm{pg} / \mathrm{mL})$ & $26.78 \pm 0.68^{\mathrm{a}}$ & $27.59 \pm 0.63^{b}$ & $28.09 \pm 0.64^{\mathrm{c}}$ & $28.96 \pm 0.69^{d}$ \\
\hline
\end{tabular}

Significant differences were obtained between seasons indicated with different letters; $(P<0.05)$; all data are presented means $\pm \mathrm{SD}$.

\section{DISCUSSION}

Serum volume decreases when small ruminants become dehydrated during water restriction. This decrease can be explained by water uptake in tissue cells (Kenneth, 2011). Hyperosmolality due to increased solute concentrations is commonly detected in water-restricted animals (Pratt et al., 2016). Reduced serum volume also increases concentrations of certain biochemical analytes, compelling animals to activate physiological mechanisms to cope with water restrictions and dehydration. In this sense, increased concentrations of biochemical analytes such as COL-T, TAG, urea, ALB, PROT-T, $\mathrm{Na}^{+}$,
CK, AVP, and Aldo in the blood of small ruminants have been considered as indicators of dehydration.

Under restricted water regimes, Jaber et al. (2004) and Ghanem et al. (2008) reported that serum COL-T concentration consistently increased in Awassi sheep (1.90 vs. 2.14 and 1.61 vs. $2.06 \mathrm{mM}$, respectively). The serum COL-T concentration also increased (47 vs. 51, 54 and $62 \mathrm{mg} / 100 \mathrm{~mL}$ with 41,31 and $21 \mathrm{~d}$, respectively) when water intake in goats $(75 \%$ German fawn and $25 \%$ Hair goat) was restricted (Kaliber et al., 2016). Vosooghi-Postindoz et al. (2018) found that the serum TAG concentration tended to 
increase when Baluchi lambs with restricted water intake were compared with groups that had free access to water (respectively $33.50 \mathrm{mg} / \mathrm{dL}$ vs. $30.50 \mathrm{mg} / \mathrm{dL}$ ). Our findings agree with these studies, as increased serum COL-T and TAG concentrations can be attributed to reduced serum volume.

Urea is a detoxifying nitrogenous compound, synthesized from ammonium ions $\left(\mathrm{NH}_{4}{ }^{+}\right)$by periportal hepatocytes, and excreted by the renal tubules to dispose of excess dietary nitrogen (Weiner et al., 2015), or recycled via salivary secretion (Jaber et al., 2004). Serum urea concentration is a good indicator of the energy intake of sheep, as it indicates the existing synchronization rate between fermentable carbohydrates and rumen degradable protein (RDP) (García et al., 2017). CK, another nitrogenous compound, is produced by myocytes and excreted by renal tubules in proportion to the muscular mass and the rate of early endogenous proteolysis in the animal (Caldeira et al., 2007).

However, under restricted water regimes, the renal function is altered with slower glomerular filtration and higher urea reabsorption rates. These can lead to increased serum concentrations of urea and CK (Weiner et al., 2015). Physiological responses of water restricted Lacaune sheep showed increased serum CK and PROT-T concentrations (Casamassima et al., 2016). Jaber et al. (2004) informed that serum urea and CK concentrations consistently increased in water deprived Awassi sheep. In another study (Abdelatif et al., 2010), waterdeprived Nubian goats also showed increased osmolality and serum concentrations of PROT-T, $\mathrm{ALB}, \mathrm{CK}$, and urea, but did not affect serum GLU concentration, as we found in our sheep.

Hormonal based response mechanisms to water deprivation consider AVP and Aldo as the key biochemical analytes in promoting water reabsorption at renal tubules, decreasing urine volume and water absorption at gastrointestinal level (Kenneth, 2011; Rotondo et al., 2016), as well as decreasing hyperosmolality as blood vessels refill with water (Ames et al., 2019). In water restricted regimes, serum AVP concentration sharply increases. Physiological responses of sheep and goat with 2 weeks of water restriction at 60 (Katahdin sheep), 50 (Boer goats), and $40 \%$ (Spanish goats) of recommended water intake, showed increased serum AVP concentrations (2.12 vs. 6.40, 7.22, and $7.06 \mathrm{pg} / \mathrm{mL}$, respectively) (Mengistu et al. 2016).

In another study where $2 \mathrm{~L}, 3 \mathrm{~L}$ and $4 \mathrm{Ld}^{-1}$ of water were restricted during 3 wk., increased serum AVP concentrations (3.47 vs. $12.9 \mathrm{pg} / \mathrm{mL}$ ) were followed by a subsequent descent (12.9 vs. $17.4,16.1$, and $14.6 \mathrm{pg} / \mathrm{mL}$ ). This drop in serum AVP concentration over time suggests that animals adapted to drinking water shortage, as this hormonal response was correlated to reductions in plasma osmolality and greater water conservation in goats $(75 \%$ German fawn and $25 \%$ Hair goat).

Finally, the mineralo corticoid Aldo is responsible for water conservation and $\mathrm{Na}^{+}$ reabsorption by renal tubules (Ames et al., 2019). In this regard, serum Aldo concentrations in Marwari sheep deprived of water increased from $18.1 \mathrm{pg} / \mathrm{mL}$ to $24.0,35.0$, and $55.0 \mathrm{pg} / \mathrm{mL}$ during 2 , 4, and $6 \mathrm{~d}$ of water restriction, respectively. A subsequent descent to a level of $18.8 \mathrm{pg} / \mathrm{mL}$ after $72 \mathrm{~h}$ of rehydration was also found (Kataria and Kataria, 2007). Climate change will compel small ruminants to become more resilient to rising environmental temperatures, and expansion of droughts (Rust, 2019). One way to attain this resilience is by selective breeding and crossbreeding programs. Therefore, populations of each species that are most resilient to warmer and dryer conditions need to be identified. Within these populations, feral sheep from Socorro Island have shown a clear seasonal variability of several biochemical analytes that indicate an adequate physiological adaptation to restricted water regimes.

\section{CONCLUSIONS}

The proposed approach of establishing reference values for different biochemical analytes in feral sheep from Socorro Island proved useful to improve the accuracy of biochemical profiling as a tool for assessment of dehydration. Calculated confidence intervals could be used at herd level to detect physiological mechanisms to cope with water restriction. Variability of reference values also opens up the possibility of selective breeding and crossbreeding programs within populations resilient to rising environmental temperatures and expanding droughts. 


\section{ACKNOWLEDGMENTS}

This research was supported by the National Council of Science and Technology-Mexico (CONACyT-Mexico) within the project "Metabolic profiles and their implications in veterinary medicine" at University of Colima. The authors wish to thank their colleagues Carlos Enrique Izquierdo-Espinal and Fernando Borderas-Tordesillas for his valued assistance.

\section{REFERENCES}

ABDELATIF, A.M.; ELSAYED, S.A.; HASSAN, Y.M. Effect of state of hydration on body weight, blood constituents and urine excretion in Nubian goats (Capra hircus). World J. Agric. Sci., v.6. p.178-188, 2010.

AMES, M.K.; ATKINS, C.E.; PITT, B. The reninangiotensin-aldosterone system and its suppression. J. Vet. Intern. Med., v.33. p.363-382, 2019.

ANTUNOVIĆ, Z.; KLIR, Z.; NOVAKOVIC, K. et al. Blood acid-base balance of Istrian goats. In: SCIENTIFIC-EXPERTS CONFERENCE OF AGRICULTURE AND FOOD INDUSTRY, 12 ., 2019, Serbia. Proceedings... Serbia: [s.n.], 2019. p.155-162. (Abstract).

BERIHULAY, H.; ABIED, A.; HE, X. et al. Adaptation mechanisms of small ruminants to environmental heat stress. Animals, v.9. p.15-21, 2019.

BERNABUCCI, U. Climate change: impact on livestock and how can we adapt. Anim. Front., v.9. p. 3-5, 2019.

CALDEIRA, R.M.; BELO, A.T.; SANTOS. et al. The effect of body condition score on blood metabolites and hormonal profiles in ewes. Small Ruminant Res., v.68. p.233-241, 2007.

CASAMASSIMA, D.; NARDOIA, M.; VIZZARRI, F. et al. The effect of waterrestriction on various physiological variables in intensively reared Lacaune ewes. Vet. Med. Czech, v.61. p.623-634, 2016.

CIANI, E.;LASAGNA, E.; D'ANDREA, M. et al. Merino and Merino-derived sheep breeds: a genome-wide intercontinental study. Genet. Sel. Evol., v.47. p.64-70, 2015.
EVANS III, E.L.; MARTÍNEZ, G.J.E.; SEHGAL, R.N.M. Phylogenetic relationships and taxonomic status of the endemic Socorro warbler (Setophaga pitiayumi graysoni). J. Ornithol., v.156. p.363370, 2015.

FLORES, P.A.; MARTÍNEZ, G.J.E.; CURRY, R.L. La vegetación de isla Socorro, archipiélago de Revillagigedo, México. Bol. Soc. Bot. Méx., v.1. p.13-23, 2009.

GARCÍA, C.A.C.; GALICIA, L.L.; BORDERAS, T.F. Reference values for biochemical analytes in mexican dairy farms: interactions and adjustments between production groups. Arq. Bras. Med. Vet. Zootec., v.69. p.445-456, 2017.

GHANEM, A.M.; JABER, L.S.; ABI-SAID, M. et al. Physiological and chemical responses in water-deprived Awassi ewes treated with vitamin C. J. Arid Environ. ,v.72. p.141-149, 2008.

HERNÁNDEZ, R.J.A.; LEPE, M.; PRADO, O. et al. Morphological study of Socorro island Merino sheep and its crosses with hair breeds. Trop. Anim. Health Prod., v.49. p.173-178, 2017.

INEGI. Colima. Geography. National Institute of Statistics, Geography and Informatics. Mexico. Available in: https://www.inegi.org.mx/. Accessed in: 30 Feb. 2020.

JABER, L.S.; HABRE, A.; RAWDA, N. et al. The effect of water restriction on certain physiological parameters in Awassi sheep. Small Ruminant Res., v.54. p.115-120, 2004.

KALIBER, M.; KOLUMAN, N.; SILANIKOVE, N. Physiological and behavioral basis for the successful adaptation of goats to severe water restriction under hot environmental conditions. Animal, v.10, p.82-88, 2016.

KANEKO, J.J.; HARVEY, W.J.; BRUSS, L.M. Appendix 7. Blood analyte reference values in large animals. In: __. (Eds.). Clinical biochemistry of domestic animals. Estados Unidos: Academic Press, 2008. p.882-888.

KATARIA, N.; KATARIA, A.K. Compartmental water management of Marwari sheep during dehydration and rehydration. Vet. Arhiv., v.77. p.551-559, 2007.

KENNETH, S.L. Water, electrolytes and acid base. In: __ (Ed.) Duncan \& Prasse's veterinary laboratory medicine. Londres: WileyBlackwell, 2011. p.145-170. 
LOTFOLLAHZADEH, S.; TEHRANI-SHARIF, M.; ZAKIAN, A.; WATSON, D.G. Assessment the alterations of some biochemical parameters in Afshari sheep with possible metabolic disorders. Small Ruminant Res., v.145. p.58-64, 2016.

MENGISTU, U.L.; PUCHALA, R.; SAHLU, T. et al. Comparison of different levels and lengths of restricted drinking water availability and measurement times with Katahdin sheep and Boer and Spanish goat wethers. Small Ruminant Res., v.144. p.320-333, 2016.

NEWSOME, D. Appropriate policy development and research needs in response to adventure racing in protected areas. Biol. Conserv., v.171. p.259-269, 2014.

ORTIZ, P.R.; CUEVA, H.; ALCANARA, J.L. et al. Avian conservation in Mexico: a 2015 snapshot. Huitzil, v.17. p.234-238, 2016.

PAYNE, J.M.; ROWLANDS, G.J.; MASTON, R. et al. A statistical appraisal of the results of the metabolic profile tests on 191 herds in the B.V.A.A.D.A.S joint exercise in animal health and productivity. Br. Vet. J., v.130. p.34-44, 1974.

PEEL, M.C.; FINLAYSON, B.L.; MCMAHON, T.A. Updated world map of the Köppen-Geiger climate classification. Hydrol. Earth Syst. Sci., v.11. p.1633-1644, 2007.

PICKERING, N.K., et al. Genetic origin of Arapawa sheep and adaptation to a feral lifestyle. Assoc Advmt Anim. Breed Genet., v.20. p.451454, 2013.

PRATT, L.R.; CHAUDHARI, M.I.; REMPE, S.B. Statistical analyses of hydrophobic interactions. $J$. Phys. Chem. B, v.120. p.6455-6460, 2016.

ROTONDO, F.; BUTZ, H.; SYRO, L.V. et al. Arginine vasopressin (AVP): a review of its historical perspectives, current research and multifunctional role in the hypothalamohypophysial system. Pituitary, v.19. p.345-355, 2016.
RUST, J.M. The impact of climate change on extensive and intensive livestock production systems. Anim. Front., v.9. p.20-25, 2019.

SALAS DE LEÓN, D.A.; GRACIA, A.; MONREAL-GOMEZ, M.A.; SALASMONREAL, D. Two years of oceanographic and meteorological data from the UNAM buoy anchored at Socorro island in the mexican pacific. Open J. Mar. Sci., v.5. p.182-192, 2015.

SOARES, G.S.L.; SOUTO, R.J.C.; CAJUEIRO, J.F.P. et al. Adaptive changes in blood biochemical profile of dairy goats during the period of transition. Rev. Méd. Vét., v.169. p.6575,2018

SOLBERG, H.E. Approved recommendation (1987) on the theory of reference values. Part 5. Statistical treatment of collected reference values. Determination of reference limits. Clin. Chim. Acta, v.170. p.S13-S32, 1987.

SPSS statistics user's guide. Version 22.0. 2013. Armonk: IBM Corp., 2013.

VAN SAUN, R.J. Indicators of dairy cow transition risks: metabolic profiling revisited. In: WORLD BUIATRICS CONGRESS, 14., 2010, Santiago. Proceedings... Santiago: [s.n.] 2010. p.65-77. (Abstract).

VOSOOGHI-POSTINDOZ, V.; TAHMASBI, A.; NASERIAN, A.A. et al. Effect of water deprivation and drinking saline water on performance, blood metabolites, nutrient digestibility, and rumen parameters in Baluchi lambs. Iran J Appl. Anim. Sci., v.8. p.445-456, 2018.

WEINER, I.D.; MITCH, W.E.; SANDS, J.M. Urea and ammonia metabolism and the control of renal nitrogen excretion. Clin. J. Am. Soc. Nephrol., v.10. p.1444-1458, 2015. 